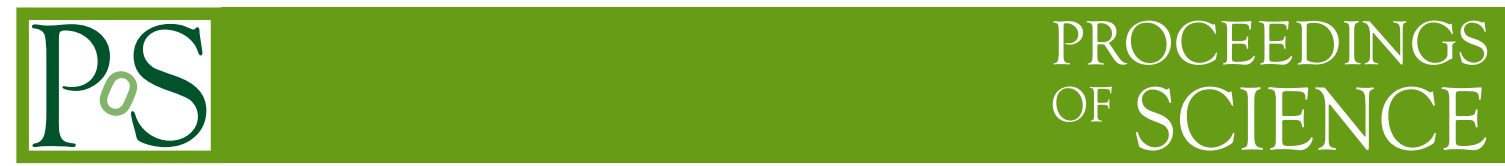

\title{
Test Beam Results of a 3D Diamond Detector
}

\author{
Marc Dünser* \\ CERN, Geneva and ETH, Zürich, both Switzerland \\ on behalf of the RD42 Collaboration ${ }^{\dagger}$ \\ E-mail: marc.dunseracern.ch
}

3D pixel technology has been used successfully in the past with silicon detectors for tracking applications. Recently, a first prototype of the same 3D technology has been produced on a chemical vapour deposited single-crystal diamond sensor. This device has been subsequently tested in a beam test at CERN's SPS accelerator in a beam of $120 \mathrm{GeV}$ protons. Details on the production and results of testbeam data are presented.

The European Physical Society Conference on High Energy Physics

22-29 July 2015

Vienna, Austria

\footnotetext{
${ }^{*}$ Speaker.

${ }^{\dagger}$ The full participant and institute list of the RD42 Collaboration is given at the end of the document
} 


\section{Introduction}

A novel detector has been fabricated which combines the already established technology of 3D [1] electrode geometry with diamond sensor material. Compared to standard planar pixel geometry which is widely employed for tracking purposes in particle physics experiments, a 3D layout profits from a much reduced drift length before collection of charge and much higher electric fields at much lower applied voltages. Diamond sensors feature an increased radiation hardness compared to silicon sensors and are thus a viable option for future applications close to the interaction point in high luminosity particle physics experiments.

The fabrication of a 3D chemical vapour deposition (CVD) diamond sensor prototype was presented including details on the fabrication, experimental setup at a testbeam at CERN's SPS accelerator [3], as well as the subsequent data-analysis. Details presented in this conference contribution are based on the work conducted for Ref. [2].

\section{Fabrication of 3D detector}

The sensor in use was a $4.7 \times 4.7 \mathrm{~mm}^{2}$ single-crystal CVD diamond of $44 \mu \mathrm{m}$ thickness [4]. Three distinct areas on the diamond were used, one with the full 3D electrode geometry and metallization, one so-called "phantom" region with identical metallization, but without the 3D electrodes, as well as a regular 2D strip-geometry for comparison. A schematic view of the different areas on the sensor material can be seen in Fig. 1 on the left hand side and a photograph of the final sensor including the wire-bonds is shown on the right of the same figure. The columns for the 3D architecture were produced by converting the isolating diamond phase into a conductive carbon phase. This was achieved by illuminating the diamond with a Ti-Sapphire femto-second laser with a wavelength of $800 \mathrm{~nm}$ with a pulse duration of $100 \mathrm{fs}$ and a repetition frequency of $1 \mathrm{kHz}$ [5]. Two grids of columns were produced, with distance parameters of $100 \mu \mathrm{m}$ and $150 \mu \mathrm{m}$, respectively. Five columns were then combined into a single "cell" with four bias columns in the corners and a readout column in the center of the cell. The efficiency of this column production was around $(92 \pm 3) \%$ as established by optical and electrical inspection. Metallization was performed with a $\mathrm{Cr}-\mathrm{Au}$ alloy and single pixels were combined into readout channels which were connected to a VA2 low-noise CMOS amplifier [6].
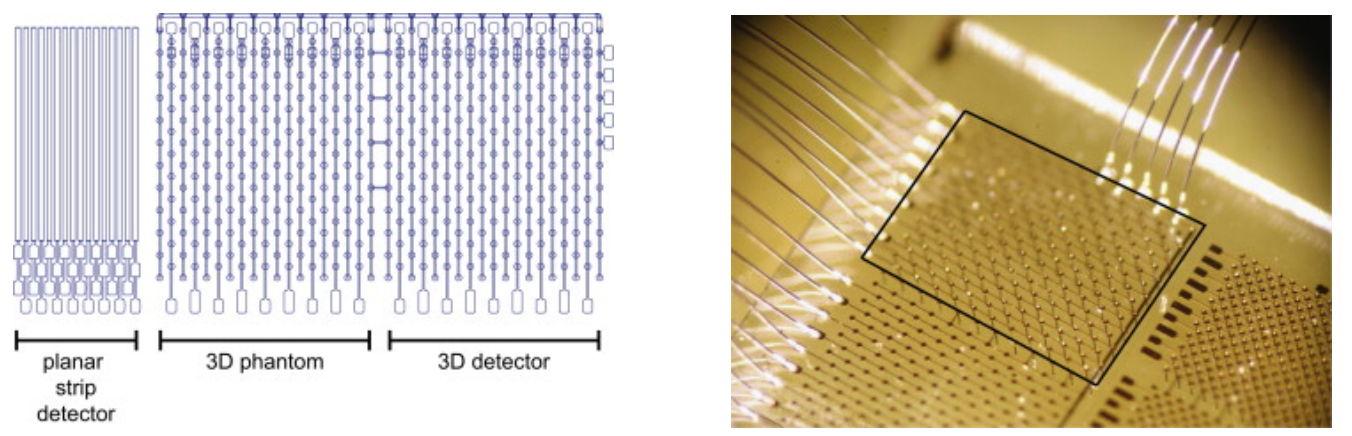

Figure 1: Schematic drawing of the prototype 3D diamond sensor and metallization (left). Photograph of the diamond sensor after metallization and wire-bonding (right). 
Source testing of the diamond sensor before metallization yielded full collection of the deposited charge at $450 \mathrm{~V}$, and the full-charge reference was measured at $500 \mathrm{~V}$ to be $(15770 \pm 250$ (stat.) \pm 350 (calib. + syst.) ) electrons. The applied voltage for the measurements in the testbeam were $500 \mathrm{~V}$ for the $2 \mathrm{D}$ strip-region and $25 \mathrm{~V}$ for the $3 \mathrm{D}$ geometry as well as the $3 \mathrm{D}$ phantom region.

\section{Testbeam and data analysis}

Testbeam measurements with high energy particles traversing the detector were performed at the H6 CERN-SPS beamline with protons at $120 \mathrm{GeV}$. For spatial resolution on the 3D detector itself, tracking was provided by a silicon tracking telescope with four times two layers of silicon strips. The final resolution of this tracking reached values of 3-5 $\mu \mathrm{m}$ on the $3 \mathrm{D}$ detector. Only events with one single track traversing the telescope were selected, corresponding to a final dataset of roughly 250000 events.

Figure 2 shows for all selected events the measured charge in the full readout planes of the diamond sensor. The 2D strip, 3D phantom, and 3D regions are marked on the two dimensional plane. It is clearly seen in the 3D detector region that some cells see much decreased signal compared to adjacent cells. This is due to faulty readout columns, while broken bias columns affect the signal collection less.

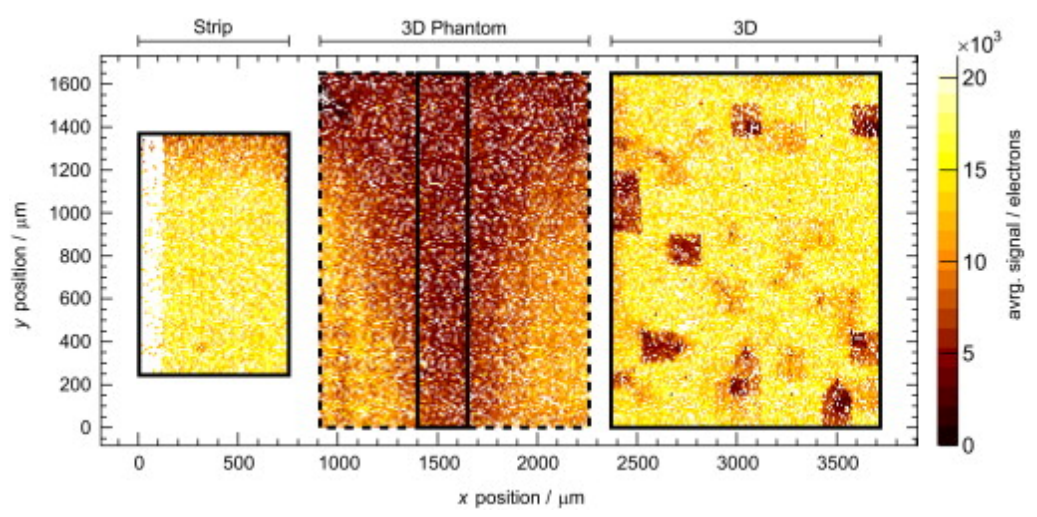

Figure 2: Measured charge on the 2D strip (left), 3D phantom (center), and 3D (right) detector geometries.

It is clearly seen from Fig. 2 that the signal collection in functioning readout cells of the $3 \mathrm{D}$ detector is similar to the charge collected in the $2 \mathrm{D}$ strip architecture, while the $3 \mathrm{D}$ phantom detector collects much less charge. The precise comparisons of the collected charge in the three different detectors are shown in Fig. 3 where the collected charge of the 3D detector is compared to the $2 \mathrm{D}$ detector (left) and to the $3 \mathrm{D}$ phantom detector (right).

The most probable value for the collected charge in the 2D planar strip detector is 13800 electrons, while the 3D geometry collects most probably 13600 electrons, indicating full charge collection at only $25 \mathrm{~V}$ compared to $500 \mathrm{~V}$ in the strip section. The 3D phantom detector, on the other hand, collects much less charge as expected, indicating it functioning only as a surface device. The most probable charge in the phantom geometry is 4400 electrons.

By restricting the measured charge to only a subset of 18 connected and functioning cells in the 3D detector, the lower peak of Fig. 3 can be removed. This is shown in Fig. 4 where the selected 

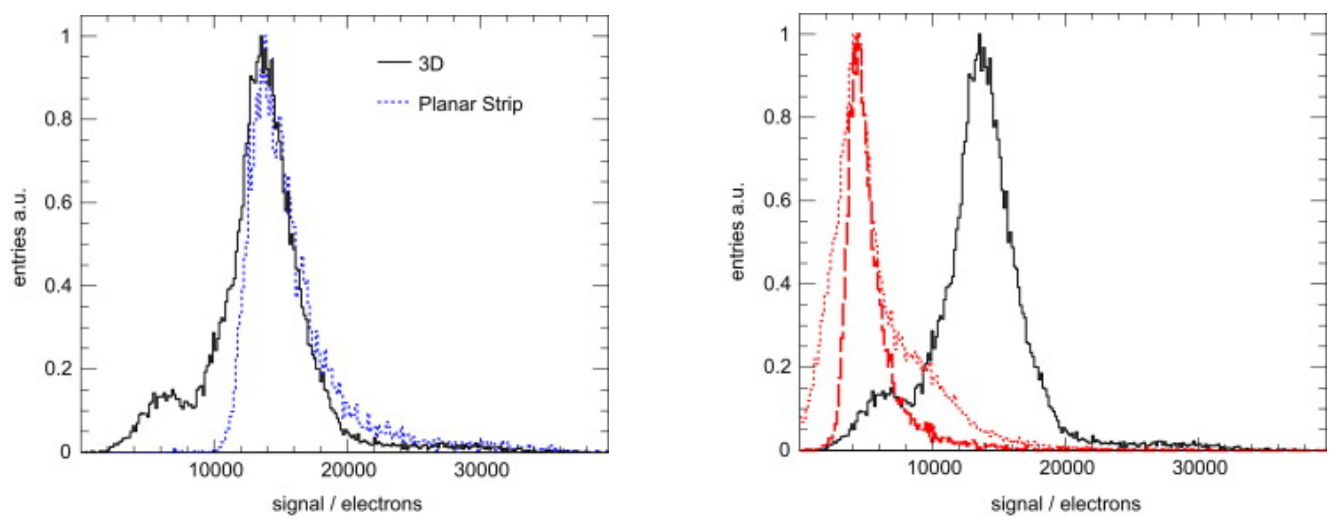

Figure 3: Comparison of measured charges in the 3D detector and 2D strip detector (left) and of the 3D detector and the 3D phantom detector (right). The 3D detector charge is shown shown as a black solid line and all distributions are scaled to the same peak height. The dashed line in the right plot indicates only the central region of the 3D phantom detector, while the broad solid line represents all charges in the 3D phantom detector.

region is indicated on the left hand side and the final collected charge, again compared to the 2D planar strip detector, is is displayed on the right hand side.
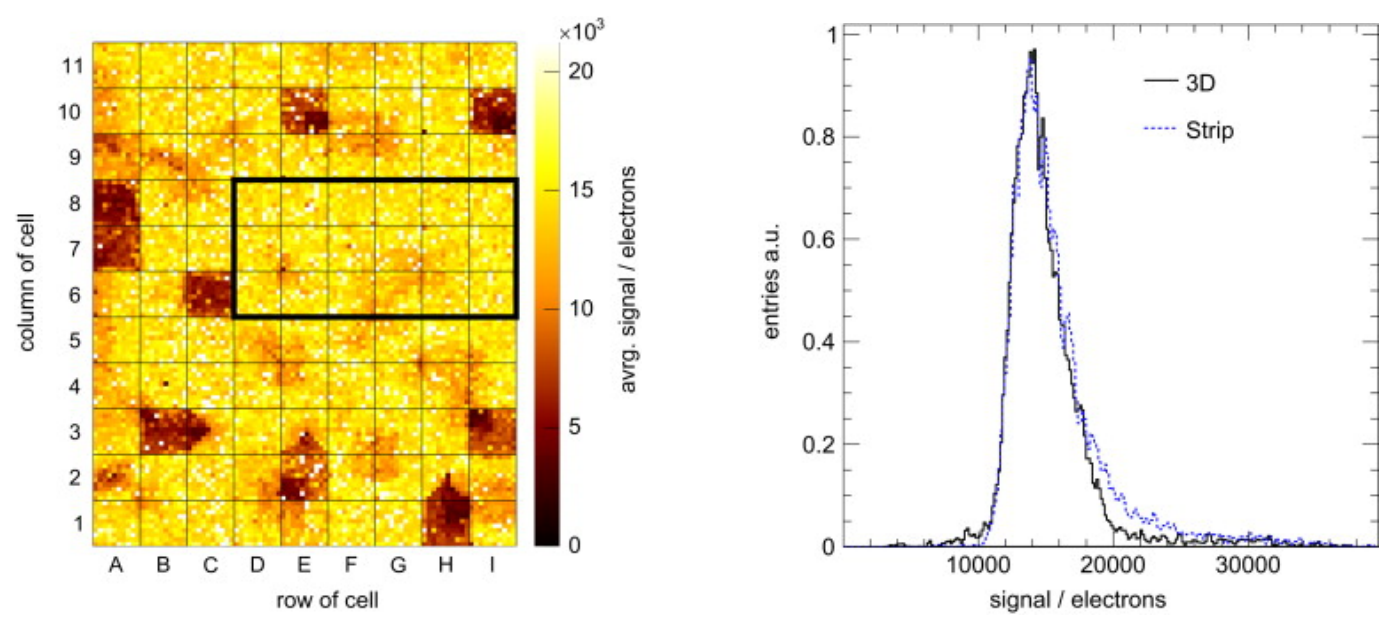

Figure 4: Selected region of the 3D detector (left) and collected charge in said region compared to the charge collected in the 2D planar strip detector (right).

Finally, the effect of the finite size of the columns themselves were investigated by looking at the average charge distribution in $10 \times 10 \mu \mathrm{m}^{2}$ sub-cells of all the 3D detector cells. This distribution is shown in Fig. 5 where the 2D distribution for an overlay of all cells and the 1D distribution are displayed. Nice agreement is found with the expected loss of charge due to a circular readout column in the center and a 4-fold shared circular bias column on the edges of each cell.

The tracking performance of the 3D diamond detector has been measured from the distribution of the residuals of traversing tracks. This distribution resembles a flat-top distribution and a 

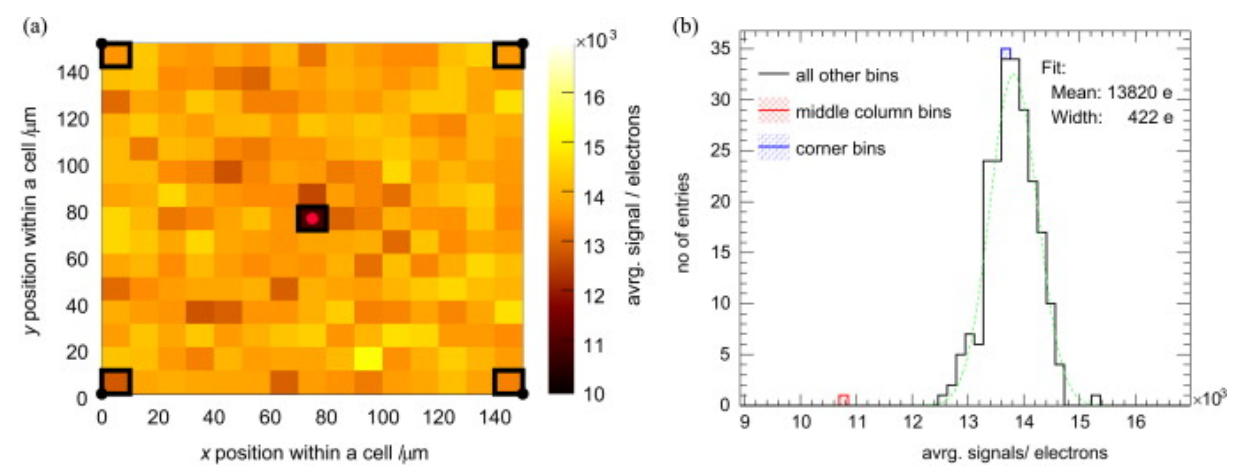

Figure 5: Overlay of the signal collection in sub-cells as a 2D distribution (right) and a 1D distribution (left).

resolution of $\sigma(x)=40.2 \pm 0.3 \mu \mathrm{m}$ is extracted, indicating no significant charge sharing between cells and reaching the digital resolution which is expected to be $43.3 \mu \mathrm{m}$.

\section{Conclusions}

The fabrication and performance of a novel 3D diamond detector for tracking applications has been presented. A testbeam campaign at the SPS at CERN was undertaken to prove the functionality of the prototype detector and to compare it to a standard 2D planar strip detector in terms of charge collection. The overwhelmingly positive results from this first prototype were presented and some details were given on the tracking performance as well, indicating that the $3 \mathrm{D}$ detector functions as expected, is capable of collecting the same charge as the standard 2D detector, and with improved column production efficiency may be studied further for future operation in high luminosity experiments.

\section{Acknowledgments}

KKG, HK, RK would like to acknowledge support from the US Department of Energy through grant DE-SC0010061. This work was partially supported by the Swiss National Science Foundation grant \#20FL20_147466 and ETH-grant 45 12-1. MD would like to thank CERN and the CMG group for financial and other support.

\section{The RD42 Collaboration}

\section{Collaboration members}

M. Artuso ${ }^{22}$, F. Bachmair ${ }^{26}$, L. Bäni2 ${ }^{26}$, M. Bartosik ${ }^{3}$, J. Beacham ${ }^{15}$, V. Bellini ${ }^{2}$, V. Belyaev ${ }^{14}$, B. Bentele ${ }^{21}$, E. Berdermann ${ }^{7}$, P. Bergonzo ${ }^{13}$, A. Bes ${ }^{30}$, J-M. Brom ${ }^{9}$, M. Bruzzi ${ }^{5}$, M. Cerv ${ }^{3}$, C. Chau ${ }^{18}$, G. Chiodini ${ }^{29}$, D. Chren ${ }^{20}$, V. Cindro ${ }^{11}$, G. Claus ${ }^{9}$, J. Collot ${ }^{30}$, S. Costa ${ }^{2}$, J. Cumalat ${ }^{21}$, A. Dabrowski ${ }^{3}$, R. D' Alessandro ${ }^{5}$, W. de Boer ${ }^{12}$, B. Dehning ${ }^{3}$, D. Dobos ${ }^{3}$, M. Dünser ${ }^{3,26}$, V. Eremin ${ }^{8}$, R. Eusebi ${ }^{27}$, G. Forcolin ${ }^{24}$, J. Forneris ${ }^{17}$, H. Frais-Kölbl ${ }^{4}$, K.K. Gan ${ }^{15}$, M. Gastal ${ }^{3}$, M. Goffe ${ }^{9}$, 
J. Goldstein ${ }^{19}$, A. Golubev ${ }^{10}$, L. Gonella ${ }^{1}$, A. Gorišek ${ }^{11}$, L. Graber ${ }^{25}$, E. Grigoriev ${ }^{10}$, J. GrosseKnetter $^{25}$, B. Gui ${ }^{15}$, M. Guthoff ${ }^{3}$, I. Haughton ${ }^{24}$, D. Hidas ${ }^{16}$, D. Hits ${ }^{26}$, M. Hoeferkamp ${ }^{23}$, T. Hofmann ${ }^{3}$, J. Hosslet ${ }^{9}$, J-Y. Hostachy ${ }^{30}$, F. Hügging ${ }^{1}$, H. Jansen ${ }^{3}$, J. Janssen ${ }^{1}$, H. Kagan ${ }^{15, \diamond}$, K. Kanxheri ${ }^{31}$, G. Kasieczka ${ }^{26}$, R. Kass ${ }^{15}$, F. Kassel ${ }^{12}$, M. Kis ${ }^{7}$, G. Kramberger ${ }^{11}$, S. Kuleshov ${ }^{10}$, A. Lacoste $^{30}$, S. Lagomarsino ${ }^{5}$, A. Lo Giudice ${ }^{17}$, C. Maazouzi ${ }^{9}$, I. Mandic ${ }^{11}$, C. Mathieu ${ }^{9}$, N. McFadden ${ }^{23}$, G. McGoldrick ${ }^{18}$, M. Menichelli ${ }^{31}$, M. Mikuž ${ }^{11}$, A. Morozzi ${ }^{31}$, J. Moss ${ }^{15}$, R. Mountain ${ }^{22}$, S. Murphy $^{24}$, A. Oh $^{24}$, P. Olivero ${ }^{17}$, G. Parrini ${ }^{5}$, D. Passeri ${ }^{31}$, M. Pauluzzi ${ }^{31}$, H. Pernegger ${ }^{3}$, R. Perrino ${ }^{29}$, F. Picollo ${ }^{17}$, M. Pomorski ${ }^{13}$, R. Potenza ${ }^{2}$, A. Quadt ${ }^{25}$, A. Re ${ }^{17}$, G. Riley ${ }^{28}$, S. Roe ${ }^{3}$, M. Sapinski ${ }^{3}$, M. Scaringella ${ }^{5}$, S. Schnetzer ${ }^{16}$, T. Schreiner ${ }^{4}$, S. Sciortino ${ }^{5}$, A. Scorzoni ${ }^{31}$, S. Seidel ${ }^{23}$, L. Servoli ${ }^{31}$, A. Sfyrla ${ }^{3}$, G. Shimchuk ${ }^{10}$, D.S. Smith ${ }^{15}$, B. Sopko ${ }^{20}$, V. Sopko ${ }^{20}$, S. Spagnolo ${ }^{29}$, S. Spanier ${ }^{28}$, K. Stenson ${ }^{21}$, R. Stone ${ }^{16}$, C. Sutera ${ }^{2}$, A. Taylor ${ }^{23}$, M. Traeger ${ }^{7}$, D. Tromson ${ }^{13}$, W. Trischuk ${ }^{18, \diamond}$, C. Tuve ${ }^{2}$, L. Uplegger ${ }^{6}$, J. Velthuis ${ }^{19}$, N. Venturi ${ }^{18}$, E. Vittone ${ }^{17}$, S. Wagner ${ }^{21}$, R. Wallny ${ }^{26}$, J.C. Wang ${ }^{22}$, P. Weilhammer ${ }^{3}$, J. Weingarten ${ }^{25}$, C. Weiss ${ }^{3}$, T. Wengler ${ }^{3}$, N. Wermes ${ }^{1}$, M. Yamouni $^{30}$, M. Zavrtanik ${ }^{11}$

\section{Institutes}

${ }^{1}$ Universität Bonn, Bonn, Germany, ${ }^{2}$ INFN/University of Catania, Catania, Italy, ${ }^{3}$ CERN, Geneva, Switzerland, ${ }^{4}$ FWT, Wiener Neustadt, Austria, ${ }^{5}$ INFN/University of Florence, Florence, Italy, ${ }^{6}$ FNAL, Batavia, USA, ${ }^{7}$ GSI, Darmstadt, Germany, ${ }^{8}$ Ioffe Institute, St. Petersburg, Russia, ${ }^{9}$ IPHC, Strasbourg, France, ${ }^{10}$ ITEP, Moscow, Russia, ${ }^{11}$ Jožef Stefan Institute, Ljubljana, Slovenia, ${ }^{12}$ Universität Karlsruhe, Karlsruhe, Germany, ${ }^{13}$ CEA-LIST Technologies Avancees, Saclay, France, ${ }^{14}$ MEPHI Institute, Moscow, Russia, ${ }^{15}$ The Ohio State University, Columbus, OH, USA, ${ }^{16}$ Rutgers University, Piscataway, NJ, USA, ${ }^{17}$ University of Torino, Torino, Italy, ${ }^{18}$ University of Toronto, Toronto, ON, Canada, ${ }^{19}$ University of Bristol, Bristol, UK, ${ }^{20}$ Czech Technical Univ., Prague, Czech Republic, ${ }^{21}$ University of Colorado, Boulder, CO, USA, ${ }^{22}$ Syracuse University, Syracuse, NY, USA, ${ }^{23}$ University of New Mexico, Albuquerque, NM, USA, ${ }^{24}$ University of Manchester, Manchester, UK, ${ }^{25}$ Universität Göttingen, Göttingen, Germany, ${ }^{26}$ ETH Zürich, Zürich, Switzerland, ${ }^{27}$ Texas A\&M, College Park Station, TX, USA, ${ }^{28}$ University of Tennessee, Knoxville, TN, USA, ${ }^{29}$ INFN-Lecce, Lecce, Italy, ${ }^{30}$ LPSC-Grenoble, Grenoble, Switzerland, ${ }^{31}$ INFN-Perugia, Perugia, Italy, $\diamond$ Spokespersons

\section{References}

[1] S. Parker, C. Kenney, J. Segel, 3D - A proposed new architecture for solid-state radiation detectors "Nucl. Inst. and Meth. in Physics Research Section A", Vol. 395 (1997), p. 328 - 343

[2] F. Bachmair et.al., A 3D diamond detector for particle tracking "Nucl. Inst. and Meth. in Physics Research Section A", Vol. 786 (2015), p. 97-104

[3] CERN, Secondary beam areas of the PS, SPS machines, 2014 website: hhtp://sba.ewb.cern.ch/sba/

[4] Element Six Ltd., Properties of electronic grade CVD diamonds, 2014

[5] Coherent Inc., Libra Series, 2014 Data Sheet (22nd Dec. 2014), website: http://www.coherent.com/downloads/LibraSeries_CoherentDataSheet_revC_May2013_4.pdf

[6] Integrated Detectors \& Electronics AS, Data Sheet VA2 Readout Chip, Oslo, Norway, 2014 